\title{
APLICACIÓN DEL PRESUPUESTO DE CAJA EN LAS MICROEMPRESAS DEL CALLAO EN EL 2013
}

\author{
CASH BUDGET FUND IMPLEMENTATION IN CALLAO MICRO-COMPANIES IN 2013 \\ Abdías Armando Torre Padilla* \\ Unidad de Posgrado de la Facultad de Ciencias Contables \\ Universidad Nacional Mayor de San Marcos-UNMSM / Lima-Perú \\ [Recepción: Febrero de 2015 / Conformidad: Marzo de 2015]
}

\section{RESUMEN}

El presupuesto de caja tiene un papel fundamental en la mejora de la capacidad económica en las micro empresas del Callao. A la luz de los cambios que son complicados y rápidos; en la actualidad, la administración de las Micro y Pequeña Empresas (MYPE) están enfrentando un reto originado por los problemas que devienen de una gestión empírica y; por tanto ineficaz, puesto que no toman en cuenta la planificación que se ve reflejada en una escasa o nula rentabilidad. En consecuencia, para contribuir a superar el problema mencionado se planteó el objetivo: Aplicar el presupuesto de caja, para mejorar la capacidad económica en las micro empresas del Callao. Luego, en el trabajo se llegó a la conclusión que a través de esta herramienta financiera, se va iniciar el camino de la mejora de su capacidad económica; y para ello, se propone el presupuesto de caja como un modelo práctico de gestión que contribuya con los resultados económicos de las microempresas.

\section{Palabras clave:}

Presupuesto de caja; capacidad económica; capacidad de competencia; crecimiento empresarial; MYPE.

\begin{abstract}
The cash budget fund has a fundamental role in improving the economic capacity in micro enterprises from Callao. In light of the changes that are complicated and fast; at present, the management of Micro and Small Enterprises (MYPE) are facing a challenge caused by problems that arise from an empirical management -and therefore ineffective- since it does not take into account the planning that is reflected in little to non-profitability. Therefore, to help overcome the problem previously mentioned, we stated this goal: applying the cash budget fund to improve the economic capacity of Callao micro-enterprises. Therefore, in our work was concluded that through this financial tool it will start on the path of improving their economic capacity; and for this, the cash budget fund is proposed as a practical management model that contributes to the economic performance of the micro-companies.
\end{abstract}

\section{Keywords:}

Cash budget; economic capacity; competition ability; business growth; MYPE.

\footnotetext{
* Maestrista en Contabilidad con Mención Banca y Finanzas. Licenciado en Administración de Empresas -UNMSM. Email: atorrepadilla@gmail.com
} 


\section{INTRODUCCIÓN}

La aplicación del presupuesto de caja como herramienta de planificación financiera en las microempresas; es un cambio que se contrapone a la costumbre tradicional, de esperar que las cosas sucedan para luego recién decidir, esta actitud del directivo ejerce presión en la inercia de la gestión. En un mundo que avanza rápidamente, solo un equipo dirigente de primer orden puede asegurar el crecimiento sostenido de una empresa.

La motivación del desarrollo del presente estudio, radica en el deseo de plantear el uso del presupuesto de caja -herramienta de planificación financiera-, que tienda a superar las barreras que generan la dirección incompetente de las microempresas que interfieren significativamente en su crecimiento. De tal forma que se logre revertir esta situación y se alcance una administración eficaz que permita mejorar su capacidad económica. Esta herramienta es importante porque suministra a la empresa una estimación de sus necesidades financieras en donde se ponen de manifiesto posibles necesidades de financiamiento o la oportunidad de utilizar los excedentes en inversiones.

Entre los problemas que se evidencian en su funcionamiento, mencionamos los siguientes:

- Carencia de conocimientos gerenciales para administrar sus negocios, por lo que adoptan una actitud empírica, que consiste en hacer de todo, centran su atención en "el día a día". No tiene tiempo para reflexionar, para hacer autocrítica. Carecen de visión a largo plazo, no planifican, no preven, no anticipan. Simplemente, reaccionan (carece de actitud estratégica); es decir, a medida que van surgiendo nuevas situaciones recién actúan, adoptando un criterio personal. Hacen de todo.

- Falta de una herramienta de planificación financiera que permita una eficaz proyección de sus ingresos y egresos, que se necesita para determinar sus necesidades financieras de corto plazo y para poder conseguir financiamiento -si fuera el casode las instituciones financieras.

- Los funcionarios de las instituciones financieras manifiestan que la mayoría de empresarios, se acercan al banco a solicitar un crédito solo con una "idea” y no con un plan que le permita saber cuáles son los principales ingresos y egresos proyectados, en qué se sustenta la información económico-financiera presentada y qué grado de seguridad de éxito tiene el negocio.

- Evidencian resistencia al cambio por temor, o porque piensan que deben aceptar lo que venga.

- Carecen de una cultura de competitividad para la mejora de la calidad de sus productos y servicios.

- Personal no competente, con poca preparación, por lo que es necesario incorporar personal capacitado.

\section{ANTECEDENTES}

De manera general, no se encontró un trabajo de especialización relacionado con el tema, empero, encontramos trabajos tangencialmente relacionados, como los siguientes:

\section{A nivel internacional:}

- Mungaray y Ramírez (2007), en el trabajo de investigación "Capital humano y productividad en microempresas”. Investigación Económica de la Facultad de Economía de la Universidad Autónoma de Baja California, México.

- García, Serrano, y Blasco (2005), en la revista de Estudios de Economía Aplicada, de la Asociación Internacional de Economía - España, publica el artículo " $i$ Competitividad e innovación en la micro y pequeña empresa? Retos previos a superar”.

\section{A nivel nacional:}

- Flores A. (2004), en su estudio para optar el grado de Doctor en la UNMSM, sobre la "Metodología de gestión para la micro, pequeñas y medianas empresas en Lima Metropolitana”.

- Duarte F. (2007), en un artículo escrito para la revista Contabilidad y Negocios de la Pontificia Universidad Católica del Perú "Emprendimiento, empresa y crecimiento empresarial”.

\section{OBJETIVO}

El objetivo de la investigación es: Aplicar el Presupuesto de Caja, para mejorar la capacidad económica en las micro empresas del Callao. 


\section{JUSTIFICACIÓN}

La investigación se justifica teóricamente porque permitirá a los microempresarios comprender mejor las finanzas, para que comprueben si han planificado en forma correcta a través del presupuesto de caja. Los gerentes podrán entonces dedicar más tiempo a los temas que hacen que las microempresas sean realmente más competentes. La investigación se justifica en forma práctica, al proporcionar un instrumento para facilitar la capacitación de los microempresarios del Callao, contribuyendo con su profesionalización en gestión financiera. De tal forma, que el microempresario capacitado podrá desempeñar mejor su labor al frente de sus MYPEs. La presentación del presupuesto de caja servirá para que el micro empresario pueda ver su posibilidad financiera y decida con fundamento técnico, la viabilidad de su inversión.

\section{MARCO TEÓRICO}

Los conceptos dentro del cual se circunscribe la investigación, son útiles para respaldarla de manera real y profunda en la actividad científica. A continuación señalaré los que tienen que ver directamente con el tema:

\section{Planificación Financiera}

Brealey y Myers (1996) describe la planificación financiera como un proceso que consiste en:

1. Analizar las alternativas de inversión y de financiación de las que dispone la empresa.

2. Proyectar las consecuencias futuras de las decisiones presentes.

3. Decidir qué alternativas adoptar.

4. Medir el resultado obtenido frente a los objetivos establecidos en el plan financiero.

\section{Presupuesto de Caja}

Es una herramienta fundamental en la planeación financiera a corto plazo. Permite al administrador financiero, identificar las necesidades y las oportunidades financieras a corto plazo. Una función de importancia del presupuesto de efectivo es ayudar al administrador a explorar la necesidad de concertar préstamos a corto plazo. La idea del presupuesto es simple: registra las estimaciones de las entradas de efectivo y salidas de efectivo. El resultado es un pronóstico de los superávit o de los déficits de efectivo.

\section{Capacidad Económica}

La capacidad económica de la empresa está dada por la condición que tiene para hacer frente a las inversiones, empleando sus fondos de manera eficiente y efectiva para alcanzar sus objetivos.

\section{Crecimiento de la Empresa}

El concepto de "crecimiento" se ha introducido en el campo empresarial desde el campo de la biología y ha pasado a significar el incremento de su capacidad productiva y de su capacidad competitiva. El crecimiento de la empresa se asocia al proceso de incremento de las operaciones (producción) a lo largo del tiempo, incluye también el de desarrollo, que se relaciona más con cambios cualitativos en el agente económico.

\section{Capacidad de Competencia}

La competitividad de las empresas tiene que ver con lo que ocurre dentro de la propia empresa: la capacidad de su gente, su nivel de desarrollo tecnológico, la calidad de sus productos, los servicios que presta, su capacidad de vinculación con otras empresas o la información de que se dispone al interior.

La capacidad de competencia son las capacidades productivas que poseen las empresas en el desarrollo de sus funciones, usando los recursos que tienen bajo ciertas condiciones, que aseguren la calidad en el logro de los resultados.

\section{METODOLOGÍA}

En esta investigación se utilizó el enfoque cuantitativo, en el cual su principal característica fue la utilización de números y la interpretación de gráficas y tablas, todo ayudado por la estadística. En cuanto al diseño es de carácter analítico explicativo. Las personas, objeto de estudio, fueron los microempresarios del Callao. La población de estudio, estuvo comprendida por 4,870 microempresas, de las cuales se obtuvo una muestra constituida por 94 microempresas. Asimismo, la investigación está circunscrita al proceso de recolección de información y la técnica empleada fue la encuesta. 


\section{RESULTADOS Y DISCUSIÓN}

La información utilizada tuvo como fuente la encuesta aplicada entre noviembre y diciembre de 2013, a la muestra de la población de MYPEs.

\section{1. ¿En qué sector de la economía se encuentra su empresa?}

Prácticamente la mayoría de los sectores elegidos por los dueños de las MYPE está concentrada en el sector servicios y el sector comercio; 55\% y $32 \%$ en ese orden, ( $87 \%$ entre ambos), probablemente porque la inversión para iniciarse en los negocios y ejercer sus actividades en estos sectores sean relativamente pequeñas. El restante $13 \%$ se ha repartido entre los sectores de manufactura con $8 \%$ y otros, con un $5 \%$.

\section{Cuadro No 01:}

Sector de la economía en donde se encuentran las MYPE del Callao

\begin{tabular}{|l|r|r|}
\hline \multicolumn{1}{|c|}{ Sector } & $\mathbf{N}^{\circ}$ de MYPEs & \% \\
\hline Servicios & 52 & 0.55 \\
\hline Comercio & 30 & 0.32 \\
\hline Manufactura & 7 & 0.08 \\
\hline Otros & 5 & 0.05 \\
\hline Total: & 94 & 1.00 \\
\hline
\end{tabular}

Fuente: Elaboración propia.

\section{En su micro empresa, ¿suelen planificar sus ingresos y egresos de efectivo?}

El resultado es que hay un $37 \%$ que sí planifica sus ingresos y egresos con presupuesto de caja; nuestra preocupación es por el 63\% (41\% que lo hace de manera muy sencilla, $16 \%$ lo hace con plan anual y $6 \%$ que no planifican), que emplean algún tipo de planificación, pero que no son precisas debido a que no están familiarizados con las estadísticas, no cuentan con registros de sus ingresos y egresos y no tienen sistematizados los aspectos interno y externo de sus negocios, los mismos que son necesarios para la planificación financiera, a ellos sumamos los que no planifican. Este grupo fue el objetivo de nuestra investigación. En la actualidad, las interrelaciones de la gestión financiera de las empresas hacen que la planificación de los ingresos y egresos de efectivo sean un paso vital en la planificación de las empresas.

\section{Considera que para garantizar el crecimiento y la eficiencia de su MYPE, ¿̇es necesaria la ca- pacitación de todo su personal incluido usted?}

Está claro que el $99 \%$; es decir, casi el 100\% está apostando por el crecimiento y la eficiencia de su negocio como consecuencia de que todos sin excepción deben estar capacitados, ellos habrían comprendido que cuanto mayor sean sus conocimientos y la de sus empleados, tanto más vigorosa y eficiente sería su organización; así mismo, habrían llegado a percibir que para garantizar el crecimiento y la eficiencia de sus MYPE, es necesario que los microempresarios tomen la decisión de motivar y facilitar la capacitación de todo su personal, e incluirse él mismo.

Bajo esta realidad reflejada en los resultados, nos permitimos reafirmar la hipótesis de la presente investigación. Y para que tengan aplicación práctica y directa, se debe capacitar a todos los actores de las MYPE, incluyendo al microempresario, no solo en esta herramienta financiera; sino también, en otras áreas de carácter administrativo y práctico en función a las necesidades de cada MYPE, a fin de que mejoren sus conocimientos con fines específicos. Al respecto, la capacitación tiene dos objetivos: mejorar la efectividad del personal de las MYPE, y mejorar los conocimientos y habilidades de gestión de los directivos propietarios básicamente en presupuesto de caja. Con la capacitación, con seguridad se garantizará sacar adelante a sus negocios y con ello, habrían logrado crecimiento y capacidad económica sostenida.

\section{Cuadro No 02:}

Sector de la economía en donde se encuentran las MYPE del Callao

\begin{tabular}{|c|r|r|r|r|r|}
\hline \multirow{2}{*}{ Suelen Planificar } & \multirow{2}{*}{$\mathbf{N}^{\circ}$} & \multicolumn{3}{|c|}{ Si } & \multirow{2}{*}{ Total } \\
\cline { 3 - 6 } & & Con presupuesto & Con plan anual & Pero, muy sencillo & \\
\hline Cantidad & 6 & 35 & 15 & 38 & 94 \\
\hline$\%$ & 0.06 & 0.37 & 0.16 & 0.41 & 1.00 \\
\hline
\end{tabular}

Fuente: Elaboración propia. 


\section{Cuadro No 03:}

Necesidad de capacitación de todo el personal y microempresario para garantizar el crecimiento y la eficiencia de las MYPE

\begin{tabular}{|c|r|r|r|}
\hline & Sí & No & Total \\
\hline Cantidad & 93 & 1 & 94 \\
\hline$\%$ & 0.99 & 0.01 & 1.00 \\
\hline
\end{tabular}

Fuente: Elaboración propia.

\section{Prueba de la Hipótesis}

La prueba de hipótesis se efectuó a través del uso de la estadística inferencial.

Hipótesis General

"La aplicación del presupuesto de caja, como herramienta de gestión de planificación financiera de corto plazo, mejora la capacidad económica en las micro empresas del Callao".

Para la confirmación de la hipótesis general de la investigación, se ha tomado del resultado de las preguntas 9 y 29 del cuestionario de la encuesta formuladas a la muestra. Así mismo, estamos suponiendo que la proporción de la población ${ }^{1}$ de MYPE del Callao que mejora su capacidad económica es de $40 \%$.

\section{Solución:}

Paso 1: Establecer la Hipótesis

$\mathrm{H}_{0}: \mathrm{P}>=0.40$ La capacidad económica alcanzada por las MYPE que aplican presupuesto de caja por lo menos es del $40 \%$.

$\mathrm{H}_{1}: \mathrm{P}<0.40$ La capacidad económica alcanzada por las MYPE que No aplican presupuesto de caja es menor del $40 \%$.

Paso 2: Determinar el nivel de significancia $\infty^{2}$

$\infty \quad=0.05=$ Nivel de significancia

$1-\infty=0.95=$ Nivel de confianza

$\mathrm{z}=-1.645=$ Valor crítico, prueba unilateral inferior
Paso 3: Calcular el valor de la estadística de prueba z

Para determinar la prueba de hipótesis, se aplica la fórmula:

$$
z=\frac{p-P o}{\sqrt{\frac{P o(1-P o)}{n}}}
$$

Donde:

$\mathrm{z}$ = Variable de prueba

$\mathrm{p}=$ Proporción muestral $=33 \%$ de MYPE que aplican presupuesto de caja $=0.33$

$\mathrm{P}_{0}=$ Proporción poblacional considerada $=40 \%$

$\left(1-\mathrm{P}_{0}\right)=$ Proporción poblacional no considerada $=60 \%$

Reemplazando los datos en la fórmula tenemos que: (z) $=-1.39$

Dado que la variable de prueba $(\mathrm{z})=-1.39$, es menor que el valor crítico $(\mathrm{z})=-1.645$, por lo que la $\mathrm{HO}$ al no pertenecer a la región critica, no se rechaza. Por consiguiente, podemos afirmar, con una confiabilidad del $95 \%$, que la capacidad económica alcanzada por las MYPE que aplican presupuesto de caja por lo menos es del $40 \%$. Este resultado lo podemos visualizar en el gráfico $\mathrm{N}^{\circ} 01$, que el valor de la estadística de prueba $\mathrm{z}$, se encuentra en la zona de aceptación de la hipótesis nula.

Paso 4: La distribución de probabilidad

Gráfico $\mathrm{N}^{\circ}$ 01: Ubicación de la variable de Prueba (z) de la H0, de la Hipótesis General

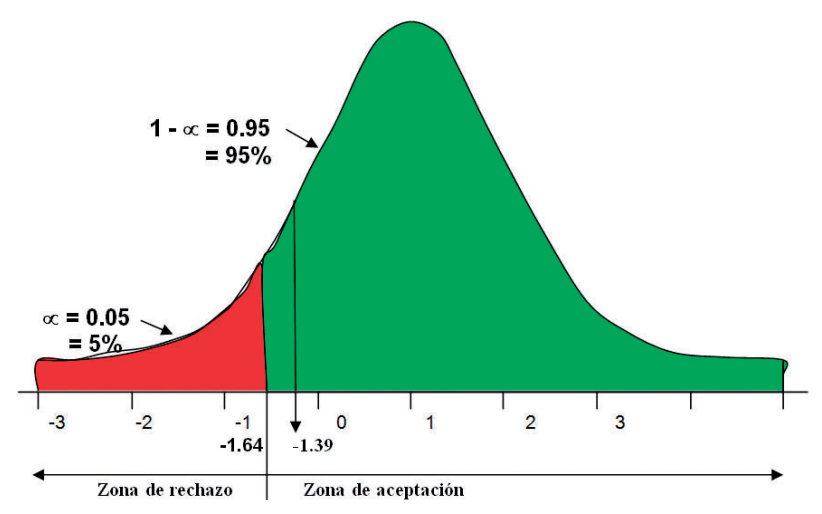

Elaboración propia.

1 Este dato generalmente es desconocido, y en las investigaciones se suelen suponer.

2 El establecimiento de $\infty$ para todos los casos, fue descrito en el proyecto de investigación. 


\section{IMPACTOS}

Dentro de la cultura de cambios el impacto es integral, alcanza a la trinidad microempresario empleados - microempresa. Lógicamente es más con el microempresario, quien es el responsable del éxito o fracaso de su negocio, en gran medida por su desempeño y, se reflejan cuando han decidido incluir la planificación financiera y aplican el presupuesto de caja como herramienta de su gestión financiera de corto plazo. Esta herramienta es utilizada para proyectar su gestión financiera de corto plazo. El impacto debe mirarse desde una perspectiva positiva, pues esta herramienta financiera debe ser la palanca que mejore la capacidad económica de sus microempresas, para que enriquezcan sus conocimientos sobre esta herramienta financiera y para que puedan hacer una gestión con mayor eficacia y así lograr el objetivo económico de la organización.

Para que tenga validez el sustento teórico y con el fin de analizar el impacto de la aplicación del presupuesto de caja en las empresas, es necesario analizar la actuación de una microempresa desde el punto de vista financiero; por esta razón -y para una mejor comprensión del valor que tiene esta herramienta de planificación financiera- procedemos a exponer su aplicación en una microempresa a través del siguiente caso práctico.

\section{CASO DE APLICACIÓN}

El caso práctico está basado en los datos obtenidos de la institución financiera como del microempresario, los mismos que de manera sistematizada se presentan a continuación:

Datos de la Institución Financiera:

La institución financiera señala que se tienen que cumplir las siguientes condiciones:

A. Como se trata de financiamiento de activos fijos, el microempresario debe aportar como mínimo el 10\% de la inversión (cuota inicial) y el $90 \%$ lo otorga el banco como financiamiento.

B. La Tasa Efectiva Anual (TEA) es de 38\%, la Tasa de Costo Efectivo Anual (TCEA) es de 41.2\%, la Tasa Mensual del Seguro de Desgravamen y, la Tasa de Seguro Multirriesgos (contra incendio).

C. Fecha de desembolso: 02 de enero del Año 01.

D. Monto de financiamiento por $S / 27,000$ y el número de cuotas de 12 (en 12 meses). La amortización mensual acorde será conforme al Cronograma de Amortización del Préstamo preparada por el banco y que a continuación se muestra:

\section{Cuadro No 01:}

Sector de la economía en donde se encuentran las MYPE del Callao

\begin{tabular}{|c|c|c|c|c|c|c|c|c|}
\hline $\begin{array}{l}\text { Nro. } \\
\text { Cuota }\end{array}$ & Fecha & Días & $\begin{array}{c}\text { Saldo } \\
\text { Inicial } \\
\end{array}$ & Amortización & Interés & $\begin{array}{c}\text { Seguro } \\
\text { Desgravamen } \\
\end{array}$ & $\begin{array}{c}\text { Seguro } \\
\text { Multirriesgo }\end{array}$ & Cuota \\
\hline 1 & $31 / 01 / 01$ & 31 & $27,000.00$ & $1,889.59$ & 759.33 & 40.34 & 9.77 & $2,699.02$ \\
\hline 2 & $28 / 02 / 01$ & 28 & $25,110.41$ & $2,019.95$ & 636.98 & 33.89 & 8.20 & $2,699.02$ \\
\hline 3 & $31 / 03 / 01$ & 31 & $23,090.47$ & $2,006.79$ & 649.38 & 34.50 & 8.35 & $2,699.02$ \\
\hline 4 & $30 / 04 / 01$ & 30 & $21,083.68$ & $2,087.60$ & 573.55 & 30.49 & 7.38 & $2,699.02$ \\
\hline 5 & $31 / 05 / 01$ & 31 & $18,996.08$ & $2,129.54$ & 534.23 & 28.38 & 6.87 & $2,699.02$ \\
\hline 6 & $30 / 06 / 01$ & 30 & $16,866.55$ & $2,209.89$ & 458.83 & 24.39 & 5.90 & $2,699.02$ \\
\hline 7 & $31 / 07 / 01$ & 31 & $14,656.65$ & $2,259.63$ & 412.19 & 21.90 & 5.30 & $2,699.02$ \\
\hline 8 & $31 / 08 / 01$ & 31 & $12,397.02$ & $2,327.37$ & 348.64 & 18.52 & 4.48 & $2,699.02$ \\
\hline 9 & $30 / 09 / 01$ & 30 & $10,069.66$ & $2,407.00$ & 273.93 & 14.56 & 3.52 & $2,699.02$ \\
\hline 10 & $31 / 10 / 01$ & 31 & $7,662.65$ & $2,469.30$ & 215.50 & 11.45 & 2.77 & $2,699.02$ \\
\hline 11 & $30 / 11 / 01$ & 30 & $5,193.35$ & $2,548.41$ & 141.28 & 7.51 & 1.82 & $2,699.02$ \\
\hline 12 & $31 / 12 / 01$ & 31 & $2,644.94$ & $2,644.94$ & 74.38 & 3.95 & 0.96 & $2,724.23$ \\
\hline \multicolumn{4}{|c|}{ TOTAL } & $27,000.00$ & $5,078.23$ & 269.89 & 65.33 & $32,413.45$ \\
\hline
\end{tabular}

Fuente: Elaboración propia. 
E. Para garantizar el correcto destino de la inversión y evitar el desvío de la inversión, el banco exige al microempresario la presentación del compromiso de compra-venta, arras o contrato de compra venta en donde estará estipulado lo siguiente: A la firma del contrato, el comprador (microempresario) paga $10 \%$ como aporte inicial y el saldo (90\%) será financiado por la entidad financiera mediante un cheque de gerencia a favor del vendedor (propietario del inmueble).

F. Además, señala que se tiene que presentar un plan de la proyección de sus ingresos y egresos a través de un Presupuesto de Caja proyectada para un período de doce meses.
Las referencias de la microempresa son las siguientes: Nombre: "Tienda: Ropa Selecta"

Establecimiento: BAZAR

Rubro: Compra y venta de ropa para damas, caballeros y niños.

Ubicación: El negocio se encuentra ubicado al frente de un mercado del distrito de Ventanilla.

Condición: Informal, actualmente se encuentra en proceso de formalización.

1. Como bien sabemos, las MYPE en su gran mayoría no llevan libros contables y tampoco manejan estados financieros. Con la información que nos brindó el microempresario, se procedió a estructurar su balance a fin de conocer cuál es su estructura patrimonial; de igual forma, procedimos a proyectar sus ventas, su costo de ventas y sus gastos operativos.

\section{Balance General al 31 de diciembre del Año 00}

\section{Efectivo}

Inventario (costo)

Muebles y enseres:

- Depreciación Acumulada

Totales
S/. 6,839.47

$24,160.53$

17,000

2,000

$\underline{15,000.00}$

$S / .46,000.00$
Cuentas por pagar $\mathrm{S} / .20,160.53$

Capital $\quad 25,839.47$

Totales S/.46,000.00

2. Resumen de las ventas anteriores y proyectadas

\begin{tabular}{|c|c|r|r|r|r|c|}
\hline Mes & \multicolumn{1}{|c|}{ Año 00 } & \multicolumn{1}{c|}{ Año 01 } & \multicolumn{1}{c|}{ Mes } & \multicolumn{1}{c|}{ Año 00 } & \multicolumn{1}{c|}{ Año 01 } & \multicolumn{1}{c|}{ Año 02 } \\
\hline Enero & $\mathrm{S} / .24,437.00$ & $\mathrm{~S} / .26,880.70$ & Julio & $\mathrm{S} / .28,276.00$ & $\mathrm{~S} / .31,103.60$ & $\mathrm{~S} / .29,568.77$ \\
\hline Febrero & $\mathrm{S} / .20,079.50$ & $\mathrm{~S} / .22,087.45$ & Agosto & $\mathrm{S} / .20,396.36$ & $\mathrm{~S} / .22,436.00$ & \\
\hline Marzo & $\mathrm{S} / .19,241.00$ & $\mathrm{~S} / .21,165.10$ & Setiembre & $\mathrm{S} / .18,433.20$ & $\mathrm{~S} / .20,276.52$ & \\
\hline Abril & $\mathrm{S} / .21,685.00$ & $\mathrm{~S} / .23,853.50$ & Octubre & $\mathrm{S} / .19,610.84$ & $\mathrm{~S} / .21,571.92$ & \\
\hline Mayo & $\mathrm{S} / .27,564.75$ & $\mathrm{~S} / .30,321.23$ & Noviembre & $\mathrm{S} / .20,078.04$ & $\mathrm{~S} / .22,085.84$ & \\
\hline Junio & $\mathrm{S} / .26,222.10$ & $\mathrm{~S} / .28,844.31$ & Diciembre & $\mathrm{S} / .55,550.56$ & $\mathrm{~S} / .61,105.62$ & \\
\hline Total & $\mathrm{S} / .139,229.35$ & $\mathrm{~S} / .153,152.29$ & & $\mathrm{~S} / .162,345.00$ & $\mathrm{~S} / .178,579.50$ & $\mathrm{~S} / .29,568.77$ \\
\hline
\end{tabular}

Se ha considerado un incremento del 10\%, para la proyección de las ventas del Año 01, y enero del Año 02 con respecto a las ventas del Año 00; de los cuales, una parte quedaría financiada con lo que se dejó de pagar por alquileres.

3. Todas las ventas se efectúan al contado, no hay nada al crédito.

4. Los sueldos y salario mensuales proyectados son de $S / .2,100.00$, con excepción de los meses de julio y diciembre en el que se debe añadir un $40 \%$ (S/.840) por concepto de aguinaldo.

5. Los alquileres mensuales del Año 00 y proyectadas son de $S / .800 .00$.

6. El Costo de Ventas del Año 00 y proyectados corresponden a un $75 \%$ de las Ventas.

7. Se requiere de un inventario mínimo de $S / .4,000$ (costo); las necesidades de mercadería (inventa- 
rio) se compran un mes antes y se pagan un mes después de efectuada la compra.

8. La empresa habrá de mantener un saldo mínimo de efectivo de S/.2,000.

9. Los gastos mensuales de los servicios (agua, luz y teléfono) no han variado, siguen en S/.500, igual al año anterior. Además, se ha previsto el $1 \%$ de las ventas para gastos imprevistos.

10. Los gastos previstos para la formalización se han estimado en S/. 2,000 los mismos que deben efectuarse en Enero.
11. La depreciación para el Año 01 -según se calculaserá de $S / .500$.

12. En el caso que fuera necesario un financiamiento adicional para atender las operaciones (menos del mínimo de caja), deberá solicitar préstamo adicional. La cantidad será en múltiplos de S/. 1,000. En el caso que el saldo fuera superior al mínimo de caja, y tuviera algún préstamo pendiente de pago, deberá proceder a utilizar los fondos excedentes para reducir la deuda. 


\begin{tabular}{|c|c|c|c|c|c|c|c|c|c|c|c|c|c|c|c|c|c|c|c|c|c|c|c|c|c|c|c|}
\hline \multirow{15}{*}{ 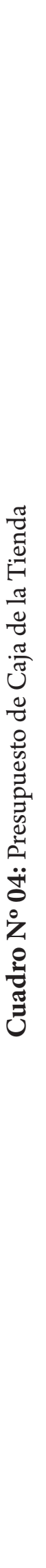 } & 嵒 & 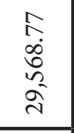 & & & & & $\begin{array}{l}\infty \\
0 \\
b \\
\vdots \\
\vdots \\
\tilde{d}\end{array}$ & & & 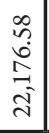 & & & & & & & & & & $\begin{array}{l}8 \\
\dot{\vec{n}} \\
\overrightarrow{2} \\
\stackrel{2}{-}\end{array}$ & & & & & & & \\
\hline & $\dot{\vec{U}}$ & $\begin{array}{l}\hat{\sigma} \\
\dot{0} \\
\stackrel{0}{-0} \\
\overrightarrow{0}\end{array}$ & & $\begin{array}{l}\hat{U} \\
\dot{0} \\
\vec{b}\end{array}$ & & 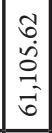 & 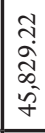 & 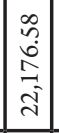 & & 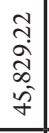 & \begin{tabular}{|l}
8 \\
0 \\
$\vdots$ \\
1 \\
$i$ \\
\end{tabular} & $\begin{array}{l}8 \\
\stackrel{0}{0} \\
\stackrel{0}{\circ} \\
\end{array}$ & & $\begin{array}{l}3 \\
0 \\
0 \\
0 \\
0 \\
\end{array}$ & $\begin{array}{l}8 \\
\stackrel{\vec{G}}{6} \\
\end{array}$ & & & 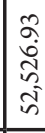 & \begin{tabular}{|c|}
0 \\
0 \\
$\infty$ \\
0 \\
$\omega$ \\
$\infty^{2}$ \\
\end{tabular} & $\begin{array}{l}n \\
0 \\
0 \\
0 \\
\\
\end{array}$ & 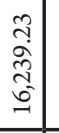 & $\begin{array}{l}8 \\
8 \\
0 \\
0 \\
i \\
\end{array}$ & 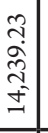 & & $\begin{array}{l}\stackrel{3}{\sim} \\
\stackrel{N}{N} \\
\stackrel{i}{i}\end{array}$ & $\begin{array}{l}8 \\
\dot{n} \\
w_{2} \\
n_{2}\end{array}$ & 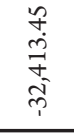 \\
\hline & $\vec{z}$ & 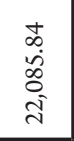 & & $\begin{array}{l}+ \\
0 \\
\dot{0} \\
\infty \\
0 \\
\text { di } \\
\end{array}$ & & 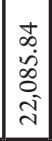 & $\begin{array}{l}\infty \\
0 \\
0 \\
0 \\
0 \\
0 \\
\\
\end{array}$ & 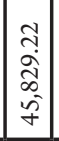 & & $\begin{array}{c}\infty \\
0 \\
0 \\
0 \\
0 \\
0 \\
-1 \\
\end{array}$ & $\begin{array}{c}8 \\
8 \\
\stackrel{8}{0} \\
-1 \\
\end{array}$ & 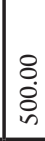 & & & 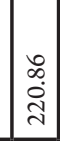 & & & 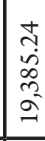 & 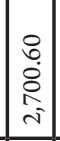 & 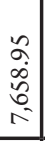 & $\begin{array}{l}\tilde{0} \\
\hat{0} \\
0 \\
0 \\
0\end{array}$ & \begin{tabular}{|c|}
8 \\
0 \\
0 \\
0 \\
$i$ \\
\end{tabular} & $\begin{array}{l}n \\
\vdots \\
\hat{\alpha} \\
\infty^{2} \\
\infty\end{array}$ & & 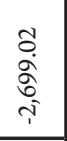 & $\begin{array}{l}n \\
0 \\
0 \\
\vdots \\
\end{array}$ & 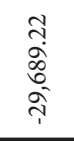 \\
\hline & ت્ટુ & 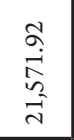 & & 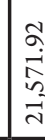 & & 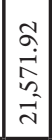 & 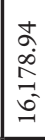 & 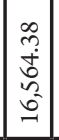 & & 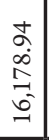 & \begin{tabular}{|c}
8 \\
0 \\
0 \\
-1 \\
\end{tabular} & $\begin{array}{l}8 \\
\stackrel{8}{\circ} \\
\dot{8}\end{array}$ & & & $\mid \begin{array}{c}\mid \\
\hat{n} \\
\\
\end{array}$ & & & 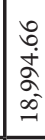 & 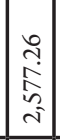 & $\begin{array}{l}r \\
\hat{0} \\
0 \\
\\
\\
\end{array}$ & $\begin{array}{l}\hat{2} \\
\hat{2} \\
\hat{2} \\
0 \\
\vdots\end{array}$ & \begin{tabular}{|c|}
0 \\
0 \\
0 \\
0 \\
$i$ \\
\end{tabular} & $\begin{array}{l}\hat{\alpha} \\
\hat{a} \\
\hat{\omega} \\
\infty^{2} \\
\infty^{2}\end{array}$ & & 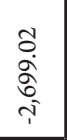 & $\begin{array}{l}n \\
\alpha \\
\infty \\
0 \\
0 \\
i\end{array}$ & 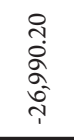 \\
\hline & 舁 & 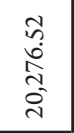 & & 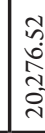 & & 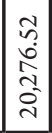 & 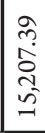 & $\mid \begin{array}{c}+ \\
0 \\
\infty \\
\stackrel{2}{2} \\
\stackrel{0}{-1}\end{array}$ & & 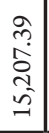 & 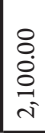 & 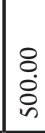 & & & $\begin{array}{l}\hat{~} \\
\text { i } \\
\text { సे }\end{array}$ & & & 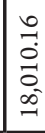 & 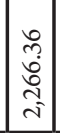 & 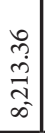 & $\begin{array}{l}0 \\
\hat{\alpha} \\
\hat{\sigma} \\
0 \\
0\end{array}$ & \begin{tabular}{|c|}
8 \\
0 \\
0 \\
0 \\
$i$
\end{tabular} & 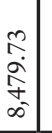 & & $\begin{array}{l}\text { ô. } \\
\text { o. } \\
\text { oे } \\
\text { i }\end{array}$ & $\begin{array}{l}\overrightarrow{1} \\
\stackrel{0}{\infty} \\
\stackrel{0}{n}\end{array}$ & 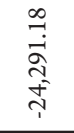 \\
\hline & $\begin{array}{l}\dot{0} \\
\dot{Z} \\
\end{array}$ & 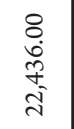 & & 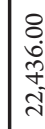 & & 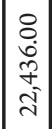 & 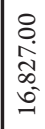 & $\mid \begin{array}{c}0 \\
\stackrel{2}{2} \\
\hat{a} \\
\hat{2} \\
\end{array}$ & & $\begin{array}{l}8 \\
\stackrel{0}{0} \\
0 \\
0 \\
0\end{array}$ & $\begin{array}{c}8 \\
8 \\
\stackrel{8}{0} \\
-1\end{array}$ & $\begin{array}{l}8 \\
\stackrel{8}{\circ} \\
\text { in }\end{array}$ & & & 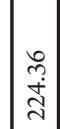 & & & 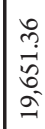 & 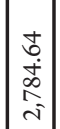 & 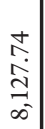 & 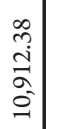 & $\begin{array}{l}8 \\
0 \\
0 \\
0 \\
i \\
\end{array}$ & 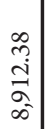 & & $\begin{array}{l}\text { ò } \\
\text { बे } \\
\text { o. } \\
i\end{array}$ & $\begin{array}{l}\infty \\
\stackrel{0}{0} \\
\vec{\pi}_{1} \\
\infty \\
\infty\end{array}$ & 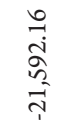 \\
\hline & s. & $\begin{array}{l}\stackrel{8}{0} \\
\stackrel{0}{0} \\
\vec{m}\end{array}$ & & $\begin{array}{l}\stackrel{8}{0} \\
\stackrel{8}{-} \\
-\overline{7}\end{array}$ & & $\begin{array}{c}0 \\
0 \\
0 \\
0 \\
= \\
m \\
\end{array}$ & $\begin{array}{l}\stackrel{2}{\curvearrowright} \\
\hat{\sim} \\
\widetilde{\sim} \\
\sim \\
\sim\end{array}$ & $\mid \begin{array}{c}8 \\
0 \\
0 \\
0 \\
0 \\
-1 \\
-1\end{array}$ & & 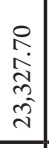 & \begin{tabular}{|l}
8 \\
0 \\
0 \\
1 \\
$i$ \\
\end{tabular} & $\begin{array}{l}8 \\
8 \\
\circ \\
\end{array}$ & & & \begin{tabular}{|l|} 
\\
$\dot{\vec{m}}$ \\
$\vec{m}$ \\
\end{tabular} & & & \begin{tabular}{|l} 
\\
0 \\
0 \\
0 \\
0 \\
id \\
\end{tabular} & 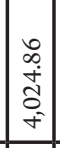 & 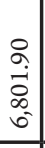 & $\begin{array}{l}0 \\
0 \\
0 \\
0 \\
0 \\
0 \\
=\end{array}$ & $\begin{array}{l}0 \\
0 \\
\dot{0} \\
\vdots \\
i \\
\end{array}$ & 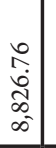 & & 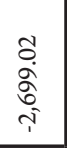 & 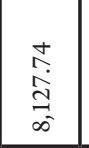 & $\begin{array}{l} \pm \\
0 \\
0 \\
\infty \\
\infty \\
\stackrel{0}{1}\end{array}$ \\
\hline & 安 & $\begin{array}{l}\vec{m} \\
\stackrel{+}{+} \\
\infty \\
\infty \\
\stackrel{0}{N}\end{array}$ & & $\begin{array}{l}\vec{m} \\
\dot{+} \\
\infty \\
\infty \\
i \\
\end{array}$ & & 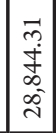 & 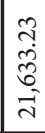 & 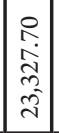 & & 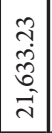 & 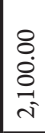 & $\begin{array}{l}8 \\
\stackrel{8}{0} \\
\dot{8}\end{array}$ & & & $\mid \begin{array}{l} \\
0 \\
\infty \\
\infty \\
i\end{array}$ & & & 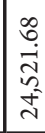 & 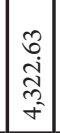 & $\begin{array}{c}\hat{c} \\
\infty \\
\stackrel{0}{c} \\
\vec{n}\end{array}$ & $\begin{array}{l}\widetilde{2} \\
\hat{0} \\
0 \\
\sigma^{2}\end{array}$ & $\begin{array}{l}8 \\
0 \\
0 \\
0 \\
i\end{array}$ & 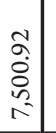 & & $\begin{array}{l}\text { Oे. } \\
\text { o. } \\
\text { i }\end{array}$ & $\begin{array}{l}\stackrel{2}{\circ} \\
\dot{0} \\
\infty \\
0\end{array}$ & 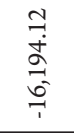 \\
\hline & 完 & 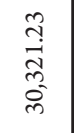 & & 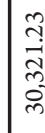 & & 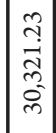 & 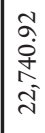 & 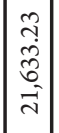 & & 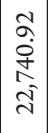 & 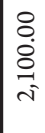 & : & & & \begin{tabular}{|l|}
$\vec{y}$ \\
$\overrightarrow{0}$ \\
0 \\
$\tilde{n}$
\end{tabular} & & & 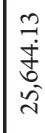 & 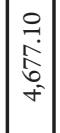 & $\begin{array}{l}\vec{c} \\
\overrightarrow{0} \\
\vec{c} \\
\vec{m}\end{array}$ & $\begin{array}{l}\overrightarrow{3} \\
\stackrel{1}{1} \\
\stackrel{\infty}{\infty} \\
\stackrel{n}{\wedge}\end{array}$ & $\begin{array}{l}8 \\
0 \\
8 \\
8 \\
i\end{array}$ & 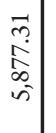 & & 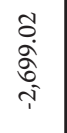 & 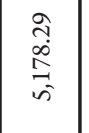 & 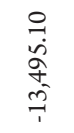 \\
\hline & $\frac{\tilde{m}}{\frac{\tilde{m}}{4}}$ & $\begin{array}{l}0 \\
0 \\
\infty \\
\infty \\
\approx \\
\sim\end{array}$ & & $\begin{array}{l}0 \\
0 \\
0 \\
\infty \\
\approx \\
\tilde{n}\end{array}$ & & 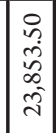 & 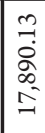 & 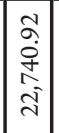 & & $\begin{array}{l}m \\
0 \\
0 \\
0 \\
\stackrel{0}{=}\end{array}$ & $\begin{array}{l}\stackrel{8}{0} \\
\stackrel{0}{0} \\
i\end{array}$ & $\begin{array}{l}8 \\
\text { कें } \\
\text { in }\end{array}$ & & & 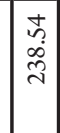 & & & 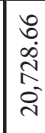 & 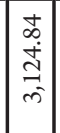 & 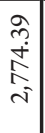 & 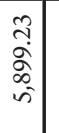 & \begin{tabular}{|c|} 
\\
0 \\
8 \\
8 \\
$i$ \\
\end{tabular} & $\begin{array}{l}\tilde{\imath} \\
\stackrel{\alpha}{\alpha} \\
\infty \\
\tilde{\infty}\end{array}$ & & $\begin{array}{l}\text { ơ. } \\
\text { Oे } \\
\text { i } \\
i\end{array}$ & $\begin{array}{l}\vec{\pi} \\
\stackrel{\Delta}{1} \\
\text { nे }\end{array}$ & 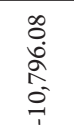 \\
\hline & $\frac{d}{\Delta}$ & $\begin{array}{l}\stackrel{0}{\vec{B}} \\
\stackrel{0}{\vec{n}} \\
\overrightarrow{\vec{N}}\end{array}$ & & 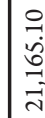 & & $\begin{array}{l}\stackrel{0}{\overrightarrow{0}} \\
0 \\
\vec{i} \\
\vec{i}\end{array}$ & 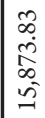 & $\mid \begin{array}{l}m \\
\vdots \\
0 \\
0 \\
0 \\
=\end{array}$ & & $\begin{array}{l}\infty \\
\infty \\
\infty \\
\infty \\
n \\
n\end{array}$ & $\begin{array}{l}8 \\
\stackrel{8}{0} \\
\stackrel{-}{v}\end{array}$ & : & & & 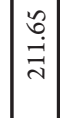 & & & 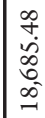 & 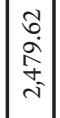 & $\begin{array}{l}\hat{\imath} \\
\hat{\alpha} \\
\hat{\imath} \\
\vec{c}\end{array}$ & 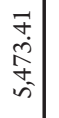 & \begin{tabular}{|c|}
8 \\
0 \\
0 \\
0 \\
$\vdots$
\end{tabular} & 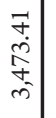 & & 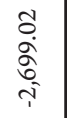 & 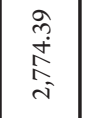 & 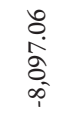 \\
\hline & 畐 & 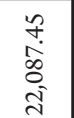 & & 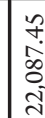 & & 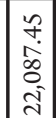 & 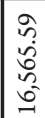 & $\left|\begin{array}{c}0 \\
\infty \\
0 \\
0 \\
0 \\
0 \\
-1\end{array}\right|$ & & $\begin{array}{l}\hat{n} \\
\hat{b} \\
\hat{0} \\
\end{array}$ & $\begin{array}{l}8 \\
8 \\
0 \\
0 \\
-1\end{array}$ & $\begin{array}{l}8 \\
8 \\
8\end{array}$ & & & $\begin{array}{l}\text { के } \\
\text { ते } \\
\vec{d}\end{array}$ & & & $\begin{array}{l}0 \\
+ \\
\infty \\
\infty \\
\sigma^{2} \\
=\end{array}$ & $\mid$\begin{tabular}{l|}
0 \\
$\hat{Q}$ \\
$\grave{2}$ \\
$\hat{i}$
\end{tabular} & 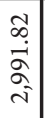 & $\begin{array}{l}\overrightarrow{0} \\
\dot{0} \\
\dot{0} \\
0 \\
n^{-}\end{array}$ & $\begin{array}{l}8 \\
8 \\
0 \\
0 \\
i\end{array}$ & $\begin{array}{c}\overrightarrow{0} \\
\dot{d} \\
0 \\
0 \\
0\end{array}$ & & 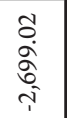 & $\begin{array}{l}\hat{\alpha} \\
\hat{\alpha} \\
\hat{i}\end{array}$ & $\begin{array}{l}+ \\
0 \\
\infty \\
0 \\
\omega^{2} \\
1\end{array}$ \\
\hline & 望 & $\begin{array}{l}R \\
\stackrel{1}{0} \\
\infty \\
\infty \\
0 \\
0\end{array}$ & & 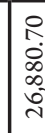 & $\mid \begin{array}{l}8 \\
0 \\
0 \\
0 \\
4\end{array}$ & 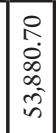 & $\begin{array}{l}n \\
0 \\
0 \\
0 \\
0\end{array}$ & $\mid \begin{array}{l}0 \\
\hat{0} \\
\hat{0} \\
\hat{0} \\
-\end{array}$ & & $\begin{array}{l}0 \\
\hat{0} \\
0 \\
0 \\
0 \\
0\end{array}$ & $\begin{array}{c}8 \\
\stackrel{0}{0} \\
\stackrel{-1}{v}\end{array}$ & $\begin{array}{l}8 \\
8 \\
\end{array}$ & $\begin{array}{l}8 \\
\\
8 \\
\text { i } \\
\end{array}$ & & 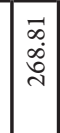 & $\begin{array}{c}8 \\
0 \\
0 \\
\vdots \\
m\end{array}$ & 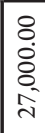 & 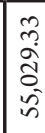 & \begin{tabular}{|c|}
0 \\
0 \\
0 \\
$\stackrel{0}{0}$ \\
-7 \\
-1
\end{tabular} & \begin{tabular}{l}
$f$ \\
\multirow{0}{*}{} \\
0 \\
0 \\
0 \\
0
\end{tabular} & $\begin{array}{l}1 \\
0 \\
0 \\
0 \\
0 \\
0 \\
0\end{array}$ & \begin{tabular}{|l|} 
\\
0 \\
0 \\
0 \\
0 \\
-1
\end{tabular} & 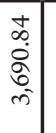 & & $\begin{array}{l}\tilde{\sigma} \\
\text { o. } \\
\text { o } \\
i\end{array}$ & $\begin{array}{l}\tilde{\alpha} \\
\dot{d} \\
\vec{\Lambda} \\
\vec{i}\end{array}$ & 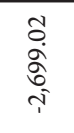 \\
\hline & ن̈ & $\begin{array}{l}0 \\
\hat{n} \\
0 \\
n \\
n \\
n\end{array}$ & & & & & 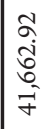 & $\mid \begin{array}{l}0 \\
0 \\
0 \\
0 \\
0 \\
0\end{array}$ & & & & & & & & & & & & & & & & & & & \\
\hline & & 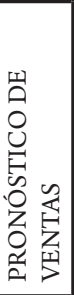 & $\begin{array}{l}0 \\
0 \\
0 \\
0 \\
0 \\
0 \\
3\end{array}$ & 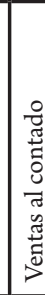 & 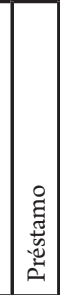 & 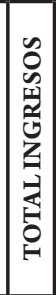 & 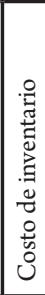 & 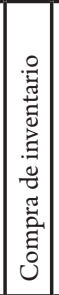 & 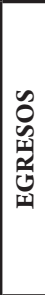 & 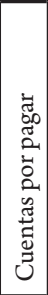 & 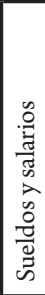 & 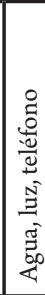 & 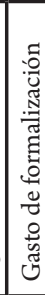 & 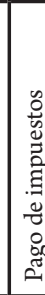 & $\mid \begin{array}{l} \\
0 \\
0 \\
0 \\
0 \\
5 \\
0 \\
0 \\
0 \\
0 \\
0 \\
0\end{array}$ & 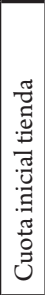 & 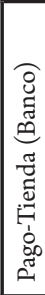 & 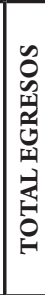 & 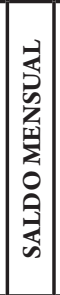 & 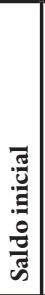 & 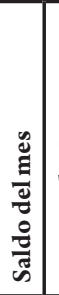 & 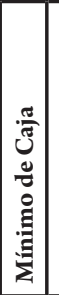 & 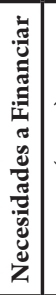 & 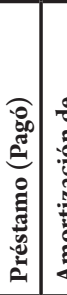 & 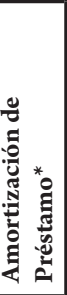 & 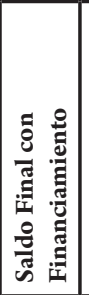 & 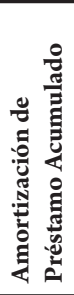 \\
\hline
\end{tabular}


Complementariamente al presupuesto de caja, se presentan los Estados Proforma. Su elaboración es importante porque dichos estados financieros nos van a reflejar la probable situación económica y financiera; así también, se podrá visualizar con mayor precisión los impactos del presupuesto de caja para la microempresa.

\section{Elaboración de los Estados Proforma y del Flujo de Fondos. ESTADO DE RESULTADOS PROFORMA}

(Al 31 de diciembre del Año 01)

Ventas

Costo de Ventas

Utilidad Bruta:

Gastos Generales y de Administración:

Sueldos y Salarios

Depreciación

Agua, luz, teléfono

Gastos de formalización

Cuota Inicial (tienda)

Amortización de Préstamo

Seguro de Desgravamen

Seguro Multirriesgo

Otros Gastos

Utilidad de Operación:

Intereses

Utilidad antes de Impuestos

Impuestos (30\%)

Utilidad después de Impuestos
$331,731.79$

$248,798.84$

$82,932.95$

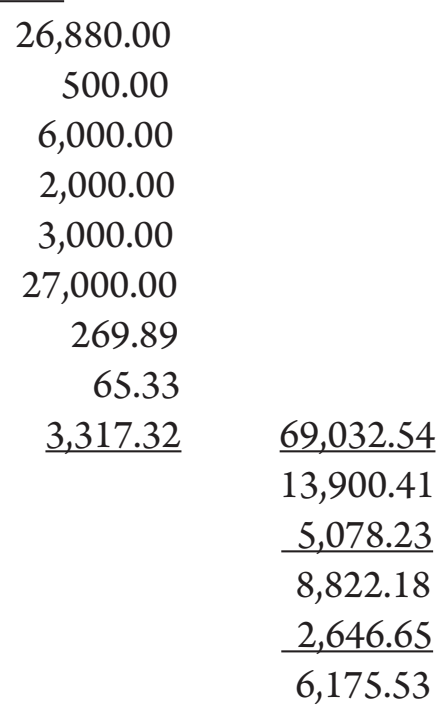

$6,175.53$

\section{Impacto Económico}

Los impactos económicos reflejados en el Estado de Resultados son los siguientes:

Ventas: Han crecido sus ventas en un $10 \%$.

Gastos Financieros: Todos los meses se va a pagar S/. 2,699.02 (con excepción de diciembre S/. 2,724.23), como amortización de la deuda.

Impuestos. Al formalizarse la microempresa ya se tendría que pagar los impuestos.

\section{BALANCE GENERAL PROFORMA}

(al 31 de diciembre del Año 01)

\section{ACTIVO}

$\underline{\text { Activo Corriente }}$

Caja

Inventario

$\underline{\text { Activo Fijo }}$

Tienda

Muebles y Enseres

(-) Depreciación

TOTAL:
$13,515.00$

$26,176.58$

$39,691.58$

$30,000.00$

$17,000.00$

$\underline{-2,500.00}$

$14,500.00$

$84,191.58$

PASIVO

Pasivo Corriente

Cuentas por Pagar

$22,176.58$

\section{Capital Permanente}

Capital 55,839.47

Utilidad del Ejercicio_ 6, 175.53

TOTAL: $\underline{62,015.00}$

$84,191.58$

128/ QVIPURAMAYOC|Vol. 23(43) 2015 


\section{Impacto Financiero}

Los impactos financieros reflejados en el balance general son los siguientes:

Caja. El disponible se reducirá en S/ 3,000 por aporte de cuota inicial de la Tienda.

Activo Fijo. El valor de los activos fijos se incrementa en $S / 30,000$

Inventarios. Los inventarios estarían financiados con las Cuentas por Pagar.

Capital. El capital se incrementa en S/ 30,000. Por lo tanto, el impacto en el patrimonio es que crece.
En el Estado de Flujo de Fondos, al comparar el balance general proforma con el balance general anterior (Dato 1) se aprecia que los activos aumentaron en $S / .38,191.58$. Este aumento deberá ser financiado por las cuentas por pagar, Capital, y por la utilidad (conforme lo refleja el incremento en dichas cuentas).

En suma, con la aplicación del caso práctico, se refuerza el marco teórico, la demostración descriptiva e inferencial, con lo que el objetivo del estudio se habría logrado.

\section{ESTADO DE FLUJO DE FONDOS DE LA TIENDA}

\begin{tabular}{|l|r|r|r|r|}
\hline \multicolumn{1}{|c|}{ ACTIVO } & \multicolumn{1}{c|}{ Año 00 } & \multicolumn{1}{c|}{ Año 01 } & Aplicación & Origen \\
\hline Activo Corriente & & & & \\
\hline Caja & $6,839.47$ & $13,515.00$ & $6,675.53$ & \\
\hline Inventario & $24,160.53$ & $26,176.58$ & $2,016.05$ & \\
\hline Activo Fijo & & & & \\
\hline Tienda & 0.00 & $30,000.00$ & $30,000.00$ & \\
\hline Muebles y Enseres & $17,000.00$ & $17,000.00$ & & \\
\hline (-) Depreciación & $-2,000.00$ & $-2,500.00$ & & 500.00 \\
\hline TOTAL: & $46,000.00$ & $84,191.58$ & & \\
\hline PASIVO & & & & \\
\hline Pasivo Corriente & & & & \\
\hline Cuentas por Pagar & $20,160.53$ & $22,176.58$ & & $2,016.05$ \\
\hline Capital Propio & & & & \\
\hline Capital & $25,839.47$ & $55,839.47$ & & $30,000.00$ \\
\hline Utilidad del Ejercicio & 0.00 & $6,175.53$ & & $6,175.53$ \\
\hline TOTAL: & $46,000.00$ & $84,191.58$ & $38,691.58$ & $38,691.58$ \\
\hline
\end{tabular}

\section{CONCLUSIONES}

Después de haber analizado el resultado y efectuado la prueba de hipótesis referente a la aplicación del presupuesto de caja para la mejora de la capacidad económica de las microempresas del Callao, se llegó a las siguientes conclusiones:

1. Les permitiría alcanzar sus objetivos que están reflejados en la mejora de su capacidad económica, lo cual evidenciaría la eficacia de las microempresas del Callao.

2. Aplicarían estrategias competitivas, que permitirían que se atienda a más clientes y las utilidades se incrementen, lo que mejoraría la capacidad de crecimiento en las microempresas del Callao.
3. Tener evidencia de manera anticipada de la situación de caja de su negocio, lo que mejoraría la capacidad económica en las microempresas del Callao.

\section{RECOMENDACIÓN}

1. Se considera que los empresarios dueños por ser los directamente implicados en la gestión de las MYPE, deben adoptar el presupuesto de caja, como herramienta de planificación financiera de corto plazo, porque su aplicación incide directamente en la mejora de la capacidad económica en su capacidad de competencia y en su capacidad de crecimiento, para que así se sientan satisfechos de la gestión que realizan. 


\section{REFERENCIAS BIBLIOGRÁFICAS}

1. BREALEY, R. A. y Myers S. C. (1996) Principios de Finanzas Corporativas. 4ta. Ed. Editorial McGraw-Hill. España.

2. DUARTE, F. (2007) Emprendimiento, empresa y crecimiento empresarial. Contabilidad y Negocios de la Pontificia Universidad Católica del Perú, vol. 2.

3. FLORES,A. (2004) Metodología de Gestión para las Micro, Pequeñas y Medianas empresas en Lima Metropolitana. Lima: UNMSM.
4. GARCÍA, J. E., SERRANO, V. C. y BLASCO, O. Ma (2005) ¿Competitividad e innovación en la micro y pequeña empresa? Retos previos a superar. Estudios de Economía Aplicada, Vol. 23. Asociación Internacional de Economía Aplicada. España.

5. MUNGARAY y RAMÍREZ (2007) Capital humano y productividad en microempresas. Investigación Económica de la Facultad de Economía de la Universidad Autónoma de Baja California. México. 